\title{
MEGAKARYOCYTIC EMPERIPOLESIS AND PLATELET FUNCTION ABNORMALITIES IN 5 PATIENTS WITH GRAY PLATELET SYNDROME
}

L.M. Larocca et al.

Supplementary information

Corresponding author:

Erica De Candia, MD, PhD, Hemostasis and Thrombosis Unit, Department of Internal Medicine, Policlinico Agostino Gemelli, Catholic University, largo Francesco Vito 1, 00168 Roma

Tel +39-06-30156329

Fax +39-06-30155915

e-mail edecandia@rm.unicatt.it 


\section{Supplemental figures}
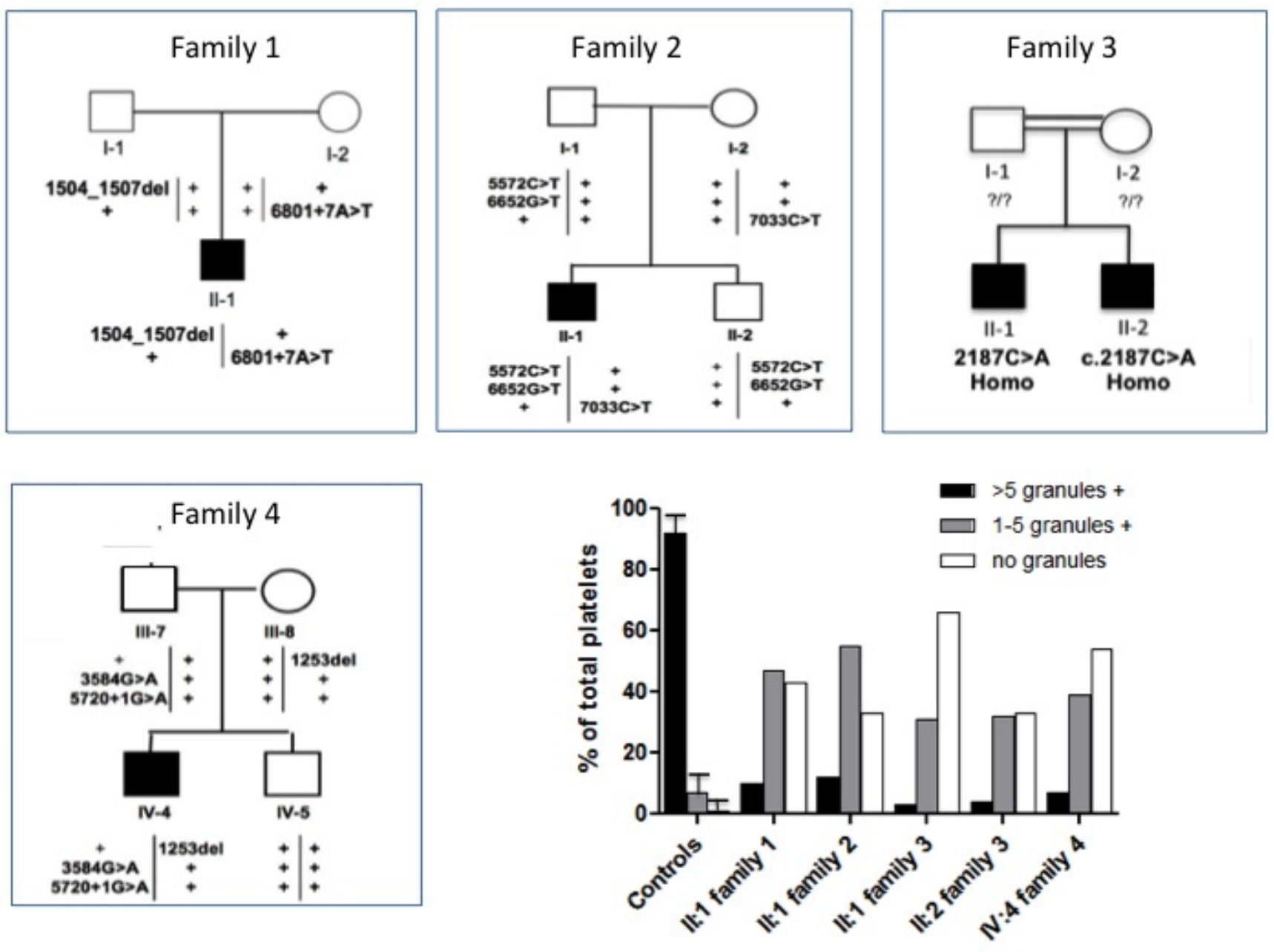

\section{Supplemental Fig. 1}

Pedigree of the families, platelet content of TSP-1 of probands

Pedigree of the four families with identified NBEAL2 mutations. Family 1 is from Barcelona, Spain; family 2 from Milan, Italy; family 3 from Buenos Aires, Argentina; family 4 from Rome, Italy.

At the time of the studies reported, the probands had the following

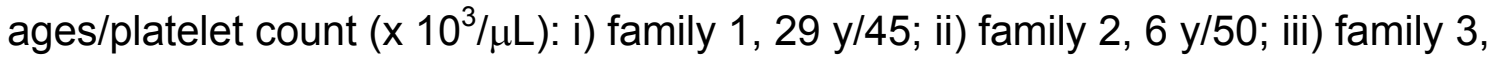
proband II:1 67 y/65 and proband II:2 72 y/30; iv) family 4, 11 y/110 (time of BM biopsy) and $21 \mathrm{y} / 60$ (time of platelet activation studies). 
The content of the $\alpha$-granule secretory protein TSP- 1 in the members of each family is expressed as $\%$ of platelets with normal ( $>5$ positive granules), moderately reduced (1-5 positive granules), severely reduced (no positive granules) content of TSP1. Results are compared to those obtained in 20 healthy controls. 

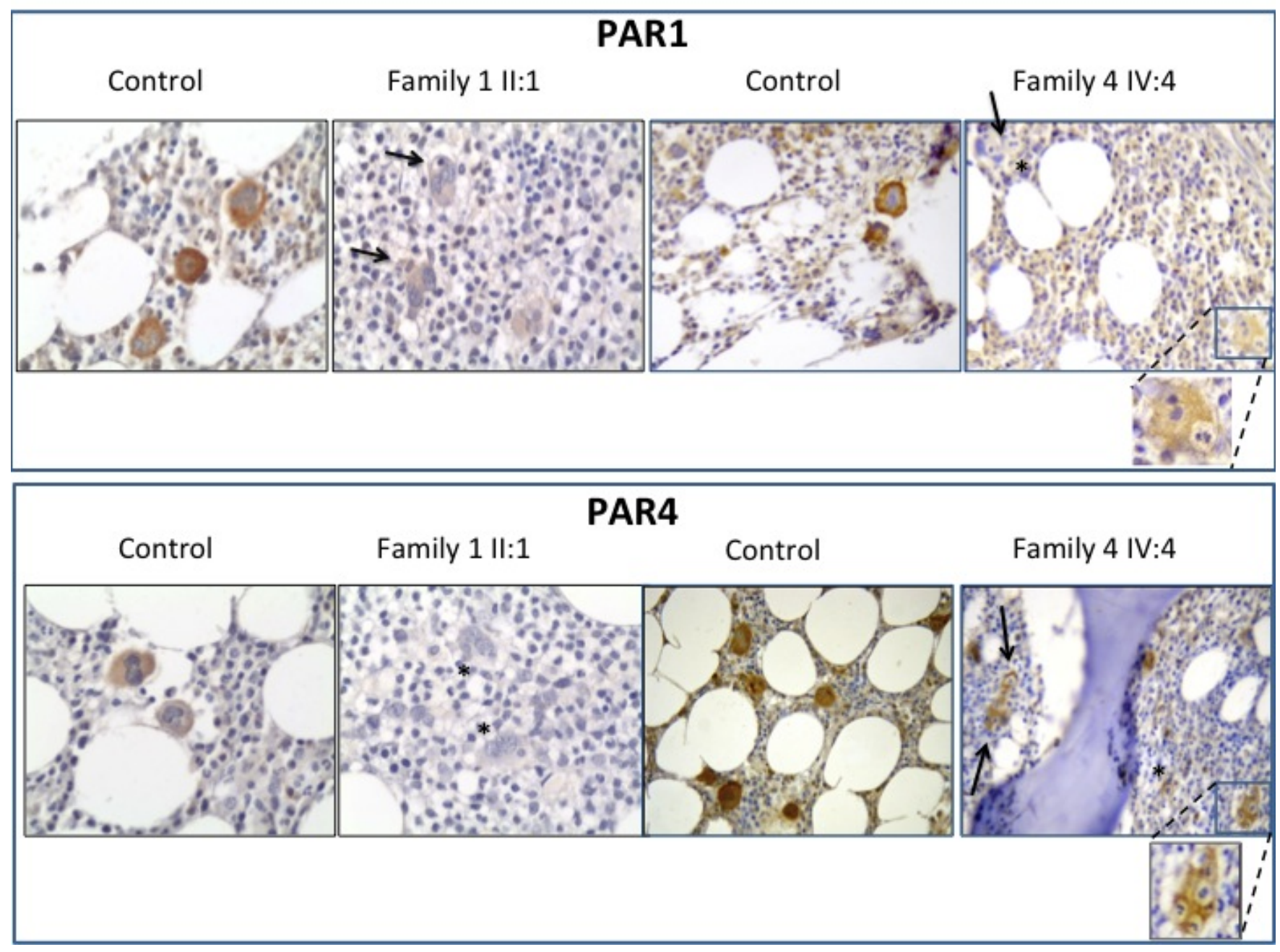

\section{Supplemental Figure 2.}

PAR1 and PAR4 content in MKs of probands of families 1 and 4.

Patients samples and controls were stained in parallel. PAR1 and PAR4 staining on GPS MKs was reduced in both patients samples compared to control, with some MKs with no staining. Arrows indicate emperipolesis.

These images are representative of 3 different slides. 


\section{Supplemental Methods}

\section{Immunofluorescence}

The content of platelet $\alpha$-granules was measured by immunofluorescence labeling of the secretory $\alpha$-granule protein thrombospondin-1 (TSP1), as previously describe. For each subject, the percentage of platelets containing i) > 5, ii) 1 to 5, iii) no TSP1 positive granules was calculate. Results obtained in patients were compared with those obtained in 20 consecutive healthy subjects who were stained in parallel. 